\title{
Densitometría ósea en niños leucémicos al completar el primer mes de quimioterapia
}

\author{
Sylvia Fischer $\mathbf{G}^{\mathbf{1}}$, Laura Neira $\mathbf{L}^{\mathbf{2}}$, Myriam Ferreiro $\mathbf{M}^{\mathbf{2}}$, \\ María Teresa Torres $C^{2}$, Vinka Giadrosich $\mathbf{R}^{2}$, \\ Aída Milinarsky $\mathrm{T}^{2}$, Marina Arriagada $\mathrm{M}^{3}$, \\ Roberto Arinoviche $S^{3}$.
}
Bone mineral density in leukemic children after completing one month of chemotherapy

Background: An important loss of bone mineral density, associated to pain and fractures, has been reported in children with acute lymphoblastic leukemia (AL). Aim: To measure bone mineral density among children with acute lymphoblastic leykemia (AL) that completed the remission induction phase with chemotherapy, that lasts 30 days. Patients and methods: children with AL, admitted to the oncology unit of a general hospital were considered eligible for the study. body composition and bone mineral density were measured by dual energy x ray absorptiometry (DEXA). each child with AU was paired with a healthly control. Results: Fourteen children age 1 to 11 years, completed the study, Spine and femoral bone mineral desities were significantly lower than in their matched controls. No differences in total body bone mineral density or content were observed. Children with ALL had a lower fat free mass and a higher fat mass than their matched controls. There was a significant correlation between fat free mass and bone mineral content. Conclusions: After one month of chemotherapy, children with AL had a lower bone mineral density in the spine and femur and a lower fat free mass (Rev Méd Chile 2005; 133: 71-76).

(Key-words: absorptionmetry, photon; bone density; Leukemia, lymphocytic, acute; Lymphoproliferative disorders)

Recibido el 15 de enero, 2004. Aceptado en versión corregida el 15 de noviembre, 2004.

${ }_{1}^{1}$ Departamento de Medicina, Escuela de Medicina, Universidad de Valparaíso.

${ }^{2}$ Departamento de Pediatría. Escuela de Medicina, Universidad de Valparaíso.

${ }^{3}$ Clínica Clinder.

$\mathrm{E}^{1}$ cáncer es la causa más frecuente de muerte en el niño mayor de 1 año, correspondiendo $1 / 3$ a leucemias ${ }^{1,2}$. La sobrevida de los niños leucémicos ha aumentado notablemente. En Chile, el Programa Infantil Nacional de Drogas Anti-

Correspondencia a: Dra. Sylvia Fischer. Hontaneda 2653 Valparaíso. Fax: 507360. E-mail: sylfisgay@yahoo.com neoplásicas (PINDA) atiende a la mayoría de los niños menores de 15 años con cáncer. La leucemia linfática aguda (LA) representa 110 casos nuevos anuales. El tercer protocolo PINDA, de 1996-1998, señala 91\% de sobrevida libre de eventos en $L A$, con una mediana de 14 meses $^{2}$ y cerca de $2 / 3$ de ellos llegan a la edad adulta ${ }^{2}$. 
Se ha descrito importante pérdida de masa ósea ${ }^{3-7}$ asociada a dolor y fracturas, en niños que han presentado LA. La pérdida de masa ósea es multifactorial, destacando: la enfermedad neoplásica misma ${ }^{4}$, la falta de ingesta de alimentos, trastornos del metabolismo de vitamina $\mathrm{D}$, el reposo debido a los periodos prolongados de hospitalización, la quimioterapia, las exacerbaciones de la enfermedad y las complicaciones ${ }^{3-12}$.

El propósito de este trabajo fue obtener mediciones de masa ósea y composición corporal, en un grupo de niños con LA, al completar la fase de inducción de la remisión con quimioterapia, que dura treinta días.

\section{PACIENTES Y MÉTODO}

Pacientes: La población estudiada incluyó a niños prepuberales que ingresaron a la Unidad Oncológica Pediátrica del Hospital Gustavo Fricke de Viña del Mar con el diagnóstico de LA, desde mayo de 2001 a enero de 2003, que aceptaron participar en el protocolo. Se excluyeron los pacientes con enfermedades que afectan el metabolismo óseo. Todos los pacientes con LIA fueron tratados según protocolo 1 del Programa Infantil Nacional de Drogas Antineoplásicas al completar la inducción de la remisión, que incluye: prednisona, vincristina, daunorrubicina, L-asparginasa y metotrexato intratecal ${ }^{13}$.

Los niños con LUA fueron pareados por edad, talla y peso con niños sanos, reclutados entre los familiares y amigos de los pacientes.

Método: Las mediciones de los pacientes se efectuaron al completar la fase de tratamiento de inducción de remisión con quimioterapia que dura 30 días, y se compararon con sus parejas controles.

Se midió la estatura en centímetros y el peso en kilogramos, en balanza marca Secca ${ }^{\circledR}$, descalzos y con ropa liviana. Se consideró el promedio de tres mediciones consecutivas efectuadas por el mismo operador.

Se realizó absorciometría radiológica de doble energía (DEXA) a los pacientes y a sus controles. Se determinó composición corporal (CC), densidad mineral ósea (DMO) en columna lumbar, cadera y cuerpo completo y contenido mineral óseo en cuerpo completo (CMO) con equipo Lunar Prodigy. El examen lo realizó un solo operador y el cuociente de variación in vitro fue $0,09 \%$, con 232 sesiones totales y el cuoficiente de variación in vivo, con 3 medidas repetidas el mismo día a niños de 3 edades diferentes fue, en columna lumbar $0,64 \%$ en cadera izquieda $0,52 \%$ y cuerpo completo $0,4 \%$. En la composición corporal, los cuocientes de variación in vivo fueron: determinación de grasa (g) 0,88\%, tejido magro (g) 0,43\%, CMO 0,97\% y masa total $0,11 \%$.

Estadística: Se demostró normalidad de las muestras con Kolmogorov-Smirnov. Se aplicó test t de Student y coeficiente de correlación de Pearson.

Esta investigación de diseño transversal descriptivo caso-control fue aprobada por el Comité de Ética del Hospital Gustavo Fricke.

\section{Resultados}

En el periodo comprendido entre mayo de 2001 y enero de 2003 ingresaron 20 niños con diagnóstico de LLA. Se excluyeron 6: 1 fallecida en el periodo de inducción, 1 lactante, por razones técnicas, 1 trasplantado de médula ósea, 1 por falta de adherencia a las indicaciones, y 2 por no lograr ser pareados con control sano. Completaron el estudio con su pareja control sano 14 niños, entre 1 y 11 años de edad. Las edades, estaturas y pesos fueron semejantes entre los 2 grupos (Tabla 1).

La DMO en columna lumbar y cadera fue significativamente menor en los niños leucémicos, con $\mathrm{p}=0,043$ y $\mathrm{p}=0,011$, respectivamente. No hubo diferencia en las determinaciones en cuerpo completo (Tabla 2).

Los niños leucémicos mostraron menos tejido magro $(p=0,033)$ y mayor porcentaje de grasa $(p=$ $0,007)$. No hubo diferencia significativa en el CMO. Aparecen espacios en blanco, correspondientes a casos en que por dificultades técnicas solo fue posible obtener algunas mediciones (Tabla 3).

Los niños leucémicos tienen una correlación $\mathrm{r}=$ 0,88 con $p=0,0007$ entre tejido magro y CMO. El grupo control sano tiene $r=0,94$ con $p<0,0001$. 
Tabla 1. Características antropométricas de pacientes con leucemia linfoblástica aguda y sus controles sanos

\begin{tabular}{|rrrrrr|}
\hline \multicolumn{2}{c}{ edad (años) } & \multicolumn{2}{c}{ talla $(\mathrm{cm})$} & \multicolumn{2}{c|}{ peso $(\mathrm{kg})$} \\
pacientes & controles & pacientes & controles & pacientes & controles \\
& & 120 & 123 & 27 & 25 \\
11,5 & 7,3 & 153 & 147,5 & 37 & 41 \\
5,9 & 10,4 & 113 & 110 & 21 & 18 \\
3,6 & 5,4 & 105 & 97 & 18,5 & 17 \\
1,7 & 3,4 & 90 & 88 & 10,5 & 14 \\
10,4 & 1,1 & 141 & 147,5 & 40,5 & 41 \\
5,4 & 10,4 & 105 & 106 & 20,5 & 20 \\
2,7 & 2,1 & 91 & 88 & 15 & 14 \\
1,8 & 2,1 & 123,5 & 130 & 10 & 14 \\
7,6 & 7,6 & 150,5 & 147 & 25 & 31 \\
11,5 & 11,5 & 110 & 110 & 43,4 & 41 \\
6 & 5,4 & 90,5 & 90 & 21,6 & 18 \\
2,8 & 2,9 & 96,7 & 97 & 15 & 12,6 \\
3,4 & 3,4 & \multicolumn{2}{c}{$\mathrm{p}=0,992$} & 13 & 17 \\
\hline
\end{tabular}

Tabla 2. D ensitometría ósea de columna lumbar, cadera y cuerpo completo en pacientes con leucemia linfoblástica aguda y controles

\begin{tabular}{|c|c|c|c|c|c|}
\hline \multicolumn{2}{|c|}{$\mathrm{DMO} \mathrm{g} / \mathrm{cm}^{2} \mathrm{~L} 2 \mathrm{~L} 4$} & \multicolumn{2}{|c|}{$\mathrm{DMO} \mathrm{g} / \mathrm{cm}^{2} \mathrm{cad}$} & \multicolumn{2}{|c|}{ DMO CC $\mathrm{g} / \mathrm{cm}^{2}$} \\
\hline pacientes & controles & pacientes & controles & pacientes & controles \\
\hline 0,541 & 0,712 & 0,577 & 0,706 & 0,789 & 0,802 \\
\hline 0,696 & 0,732 & 0,877 & 0,798 & 0,86 & 0,959 \\
\hline 0,58 & 0,586 & 0,577 & 0,718 & 0,79 & 0,807 \\
\hline 0,493 & 0,562 & 0,504 & 0,697 & 0,712 & 0,794 \\
\hline 0,391 & 0,542 & 0,32 & 0,574 & & \\
\hline 0,735 & 0,732 & 0,748 & 0,798 & 0,94 & 0,959 \\
\hline 0,596 & 0,687 & 0,568 & 0,706 & 0,696 & 0,766 \\
\hline 0,472 & 0,542 & 0,467 & 0,574 & & \\
\hline 0,454 & 0,542 & 0,427 & 0,574 & & \\
\hline 0,597 & 0,602 & 0,785 & 0,682 & 0,837 & 0,721 \\
\hline 0,889 & 0,732 & 0,934 & 0,798 & 0,935 & 0,959 \\
\hline 0,679 & 0,586 & 0,655 & 0,718 & 0,887 & 0,807 \\
\hline 0,45 & 0,505 & 0,517 & 0,556 & 0,726 & 0,7 \\
\hline 0,422 & 0,562 & 0,422 & 0,697 & & \\
\hline
\end{tabular}


Tabla 3. Composición corporal en pacientes con leucemia linfoblástica aguda y controles

\begin{tabular}{|ccrrrr|}
\hline \multicolumn{2}{c}{ \% grasa } & \multicolumn{2}{c}{ magro (g) } & \multicolumn{2}{c|}{ CMO (g) } \\
pacientes & controles & pacientes & controles & pacientes & controles \\
\hline 34,6 & 23,3 & 16,99 & 17,9 & 735 & 869 \\
22,1 & 32,5 & 27,36 & 26,6 & 1512 & 1530 \\
34,2 & 10,8 & 13,6 & 15,063 & 694 & 629 \\
24,7 & 25,5 & 13,25 & 11,979 & 477 & 512 \\
46,7 & 32,5 & 20,6 & 26,6 & 1299 & 1530 \\
38,3 & 25 & 11,9 & 14,4 & 500 & 586 \\
26,4 & 23,6 & 17,6 & 23,3 & 900 & 774 \\
46,3 & 32,5 & 20,1 & 26,7 & 1700 & 1530 \\
22,7 & 10,8 & 15,5 & 15,1 & 761 & 629 \\
26,5 & 16,2 & 10,9 & 10,3 & 397 & 356 \\
\hline
\end{tabular}

$\mathrm{p}=0,007$

$p=0,033$

$\mathrm{p}=0,471$

\section{DiscUSIÓN}

Los resultados mostraron que el grupo de niños con LAA estudiado, al completar la fase de inducción de la remisión con quimioterapia, que dura treinta días, tiene menos DMO a nivel de columna lumbar y cadera y normalidad en cuerpo completo. El estudio fue realizado en niños prepuberales, lo que evita la influencia de la maduración sexual sobre el desarrollo del esqueleto ${ }^{14}$ y fueron comparados con niños sanos de estatura y peso similares, con lo que estimamos se minimiza el efecto del tamaño del hueso sobre la densidad areal y volumétrica en los valores de DMO ${ }^{15}$.

Por razones éticas, que impedían postergar el inicio del tratamiento, fue necesario esperar a que se completara la fase de inducción de la remisión antes de someterles a la evaluación densitométrica, al no disponer del equipo dentro del recinto hospitalario. La evaluación de la masa ósea de nuestros pacientes fue realizada al completar un mes de quimioterapia.

La terapia antineoplásica incluye corticoides con dosis acumulativa aproximada de prednisona de $1.800 \mathrm{mg} / \mathrm{m}^{2}$ y metrotrexato, ambos medicamentos, reconocidamente, afectan el metabolismo óseo, aumentando la resorción e inhibiendo la actividad osteoblástica ${ }^{4,6,9-12}$.
$\mathrm{Si}$ bien es ampliamente reconocido el efecto deletéreo del corticoide en dosis farmacológicas y prolongadas sobre la masa ósea, al revisar la literatura no se describen cambios densitométricos objetivables al cabo de 1 mes de tratamiento, por lo que tenemos duda si la menor masa ósea pueda estar en parte influída ya tan tempranamente por esta terapia.

Los mecanismos mediante los cuales los corticoides pudieran afectar el metabolismo óseo son variados. El uso de corticosteroides y otros agentes antineoplásicos produce un aumento de la utilización y un trastorno de la síntesis de la $1,25(\mathrm{OH})_{2}-\mathrm{D}^{6}$. Por otro lado, los corticoides provocan una disminución directa de la actividad osteoblástica con disminución de su número absoluto y apoptosis prematura ${ }^{21}$. La quimioterapia afecta el eje de la hormona del crecimiento, con consecuencias sobre el metabolismo óseo ${ }^{18}$.

La leucemia afectaría el metabolismo óseo en forma independiente de la terapia. Hay discrepancia en los hallazgos de la masa mineral ósea al inicio de la enfermedad. La menor DMO encontrada en columna lumbar y cuello femoral concuerda con lo descrito por otros autores ${ }^{5,7}$. Boot ${ }^{16}$ refiere menor DMO sólo en algunos pacientes al diagnóstico de LA, Arikoski ${ }^{3}$ en cambio, no demostró menor DMO en los niños con LAA al momento del diagnóstico. 
Aunque en columna lumbar y cadera hubo menor DMO, la normalidad descrita en la DMO de cuerpo completo puede ser explicada porque columna y cuello femoral tienen alta proporción de hueso trabecular, de más rápido recambio y que corresponde sólo al 15\% del esqueleto ${ }^{14}$.

Se han descrito diferentes mecanismos por los que la neoplasia puede afectar el metabolismo óseo: la neoplasia misma, el compromiso nutricional, el reposo y el tratamiento.

En los procesos neoplásicos, se señalan efectos sobre el metabolismo óseo mediados por péptidos similares a PTH, citoquinas secretadas por las celulas tumorales, trastornos en la secreción de hormona del crecimiento, trastornos del metabolismo de la vitamina $\mathrm{D}$ e infiltración leucémica de la médula ósea desde la etapa inicial de la enfermedad neoplásica ${ }^{3,5,6}$. La osteopenia en niños con leucemia ha sido asociada con alteraciones del metabolismo de la vitamina $\mathrm{D}^{5,6}$. Halton observó que la mayoría de los niños con LA tenían bajas concentraciones plasmáticas de 1,25$(\mathrm{OH})_{2} \mathrm{D}$ al momento del diagnóstico, y estos niveles permanecían bajos durante la terapia, mientras que los niveles de 25 OH-D fueron normales 5 .

La 1,25(OH) $)_{2}$-vitamina $\mathrm{D}$ actúa como una hormona que estimula la absorción intestinal de calcio y fósforo, la resorción ósea y la reabsorción tubular renal de calcio. También es responsable de la síntesis de osteocalcina ${ }^{5}$. Se ha reportado ${ }^{3,5}$ la posibilidad que la $1,25(\mathrm{OH})_{2}$-vitamina $\mathrm{D}$, se una a su receptor específico en las células leucémicas, y esto aumente sus requerimientos antes que las células de la línea mieloide puedan diferenciarse.

\section{REFERENCIAS}

1. RizzaRdini M. Pediatría. Editorial Mediterráneo. Santiago de Chile. 1999, página 631.

2. Vargas L Cáncer en Pediatría. Aspectos generales. Rev Chil Pediatr 2000; 71(4): 283-95.

3. Arikoski P, Komulainen J, Riikonen P, Voutilainen R, KNIP, M KRoGeR H. Alterations in Bone Turnover and Impaired Development of Bone Mineral Density in Newly Diagnosed Children with Cancer: a 1 year Prospective Study. J Clin Endocrinol Metab 1999; 9: 3174-81.
La adecuada nutrición e ingesta de calcio son fundamentales en la normalidad del metabolismo óseo ${ }^{14}$. La menor ingesta alimentaria en los niños con LLA, en esta fase, se debe a la anorexia, que es un síntoma precoz y es exacerbado por la quimioterapia y las eventuales infecciones ${ }^{8-10}$. Siendo la LA de inicio agudo (días), es poco probable que la menor masa ósea observada al diagnóstico pueda atribuirse en tan corto tiempo al factor nutricional, aunque sí tendrá importancia en la evolución de estos niños.

Los niños con LA disminuyen su actividad física por el compromiso del estado general que le provoca la enfermedad y por la hospitalización para efectuar esta primera fase de tratamiento. Se ha establecido la importancia del ejercicio para mantener e incrementar la masa ósea ${ }^{19,20}$, así como el impacto negativo del reposo prolongado sobre la masa ósea.

Los niños leucémicos mostraron menos tejido magro $(p=0,033)$ y mayor porcentaje de grasa $(p=$ 0,007 ). En estos pacientes se ha descrito significativa menor masa magra al momento del diagnóstico, sin diferencias en el porcentaje de masa grasa al compararlos con controles sanos ${ }^{22}$. Nuestros resultados incluyen, además, el efecto de 1 mes de quimioterapia. Van der Sluis ${ }^{22}$ refiere progresivo aumento del porcentaje de grasa corporal desde las primeras semanas de quimioterapia y lo atribuye fundamentalmente al corticoide. En niños tratados por LA hubo exceso de ganancia de peso no relacionada con el gasto metabólico de reposo ${ }^{23}$.

En conclusión, los niños leucémicos, al completar el primer mes de tratamiento, muestran menor DMO en columna lumbar y cadera. Tienen menos tejido magro y mayor porcentaje de grasa.

4. Arikoski P, Koumulainen JT, Riikonen P, Jurveun J, Voutilainen RJ, KROger H. Reduced bone density at completion of chemotherapy for a malignancy. Arch Dis Child 1999; 80: 143-8.

5. Halton JM, Atkinson SA, Fraher L, Webber CE, COCKSHOTT WP, TAM C, BARR RD. Mineral homeostasis and bone mass at diagnosis in children with acute lymphoblastic leukemia. J Pediatr 1995; 126: $557-64$.

6. Halton JM, Atkinson SA, Fraher L, Webber C, Giш GJ, DAwSON S, BARR RD. Altered mineral metabolism and bone mass in children during treatment 
for acute lymphoblastic leukemia. J Bone Miner Res 1996; 11: 1774-83.

7. Van Der Sluis I, Van Der Heuvel-Eibrink M, HäHlen K, Krenning E, De Muinck Keizer-Schrama S. Altered bone mineral density and body composition, and increased fracture risk in childhood acute lymphoblastic leukemia. J Pediatr 2002; 141: 20410.

8. Henderson RC, Madsen CD, Davis C, Gold SH. Bone density in survivors of child malignancies. J Pediatr Hematol Oncol 1996; 18: 367-72.

9. Henderson RC, Madsen CD, Davis C, Gold SH. Longitudinal evaluation of bone mineral density in children receiving chemotherapy. J Pediatr Hematol Oncol 1998; 20: 322-6.

10. SchWarTZ AM, Leonidas JC. Methrotrexate osteopathy, Skel Radiol 1984; 11: 13-6.

11. SAAG KG. Glucorticoid-induced osteoporosis. Endocrinol Metab Clin North Am 2003; 32: 135-57.

12. Campbel M. Protocolo Programa Infantil Nacional Drogas Antineoplásicas. Ministerio de Salud 1996.

13. Mora S, GiLsanz V. Establishment of peak bone mass. Endocrinol Metab Clin North Am. 2003; 32: 39-63.

14. SEEMAN E. The structural and biomechanical basis of the gain and loss of bone strength in women and men. Endocrinol Metab Clin North Am 2003; 32(1): 25-38.

15. Boot AM, Van Den Heuvel-Eibrink MM, Hählen K, Krenning EP, De Muinck Keizer-Schrama SMPF. Bone mineral density in acute lymphoblastic leukaemia. Eur J Cancer 1999; 35: 1693-7.

16. SOYKa L, FAIRFIELD W, KLibansKi A. Hormonal determinants and disorders of peak bone mass in children. J Clin Endocrinol Metabol 2000; 85: 3951-65.
17. Crofton PM, Ahmed SF, Wade JC, Stephen R, Elmunger MW, Ranke MB, Kelnar CJ, WaLace WH. Effects of intensive chemotherapy on bone and collagen turnover and the growth hormone axis in children with acute lymphoblastic leukemia. J Clin Endocrinol Metab 1998; 83: 3121-9.

18. Timmann V, Darington AS, Eiser C, Bishop NJ, Davies HA. Male sex and low physical activity are associated with reduced spine bone mineral density in survivors of childhood acute lymphoblastic leukemia. J Bone Miner Res 2002; 17(6): 1073-80.

19. MinAIRE P. Inmobilization osteoporosis: a review. Clin Rheumatol 1989; 8 (suppl 2): 95-103.

20. Canals E. Mechanisms of glucocorticoid action in bone. Implications to glucocorticoid-induced osteoporosis. J Clin Endocrinol Metabol 1996; 81: 3441-7.

21. Van Der Sluis IM, Van Den Heuvel-Eibrink MM, Hahlen K, Krenning EP, DE Muinck Keizer-Schrama SM. Altered bone mineral density and body composition, and increased fracture risk in childhood acute lymphoblastic leukemia. J Pediatr 2002; 141: 204-10.

22. Reily JJ, Blacklock CJ, Daie E, Donaldson M, Gibson BE. Resting Metabolic Rate and Obesity in Childhood Acute Lymphoblastic Leukaemia. Int J Obes Relat Metab Disord 1996; 20(12): 1130-2.

23. Boot AM, Bouquet J, De Ridder MAJ, Krenning EP, De Muinck KeIZer-Schrama SMPF. Determinants of body composition, measured by dual X-ray absorptiometry, in Dutch children and adolescents. Am J Clin Nutr 1997; 66: 232-8.

Agradecimientos:

Los autores agradecen a Gabriela González por su ayuda como secretaria y a María Paz Casanova por su asesoría estadística. 